\title{
¿CÓMO REINTERPRETAR LA REGULACIÓN DE LA PROPIEDAD EN EL CÓDIGO CIVIL DESDE LOS ESTÁNDARES DEL INDIVIDUALISMO, SIMPLIFICACIÓN Y ABSTRACCIÓN?*
}

How to reinterpret the regulation of property on the Civil Code

\author{
FROM THE STANDARDS OF INDIVIDUALISM, SIMPLIFICATION AND ABSTRACTION? \\ COMMENT REINTERPRETER LA REGULATION DE LA PROPRIÉTÉ \\ Dans le Code Civil depuis les STANDARdS DE L'individualisme, \\ LA SIMPLIFICATION ET L'ABSTRACTION?
}

Jaime Manríquez Oyanader**

\begin{abstract}
RESUMEN
El artículo se aboca a la tarea de reinterpretar la concepción de la propiedad privada desde el Código Civil, que carece de contenido normativo o justificativo, a partir de los estándares conexos de individualismo, abstracción y simplificación, atingentes a una construcción decimonónica, pero que no alcanzarian su completitud sino hasta la obra pandectista, y por tanto, se construye bajo la premisa de que Bello sería, al igual que Portalis, un hombre de frontera en su concepción de propiedad. Se recurrirá a elementos dogmáticos, normativos e históricos tendiente a dar cuenta de la pugna planteada y no resuelta por el codificador entre libertad e igualdad, que permite reconstruir la propiedad en un coherente sistema tanto en su faz subjetiva como objetiva.
\end{abstract}

PALABRAS CLAVE: Propiedad privada - Abstracción - Individualismo - Simplificación

ABSTRACT

The article tackles the task of reinterpreting the concept of private property from the Civil Code which lacks normative or justificatory content, based on related standards of individualism, abstraction and simplification, pertaining to a construct of the nineteenth century, but that will not reach their completeness until the pandectist work, and therefore is built on the premise that Bello would be as well as Portalis, a border man in his conception of property. Dogmatic, normative and historical elements will be used in order to account for the proposed conflict, not solved by the encoder, between liberty and equality, which allows the rebuilding the notion of property into a coherent system both in its subjective and objective aspect.

KEYWORDS: Private property - Abstraction - Individualism - Simplification

RÉSUMÉ

L'article vise à réinterpréter le concept de la propriété privée depuis le Code Civil, lequel manque de contenu normatif ou justificatif, en partant des standards connexes de l'individualisme, la

\footnotetext{
*Artículo recibido el 29 de octubre de 2015 y aceptado para su publicación el 4 de diciembre de 2015.

** Abogado. Licenciado en Ciencias Jurídicas por la Universidad Católica de Temuco. Estudiante Magíster en Derecho con y sin menciones Facultad de Derecho, Universidad de Chile. Correspondencia a: Correo electrónico jmanri.oyanader@gmail.com.

Agradezco los valiosos comentarios que recibí del profesor Esteban Pereira Fredes sobre un borrador de este artículo.
} 
simplification et l'abstraction. Ces derniers appartiennent à une construction du dix-neuvième siècle, néanmoins, ils n'atteignent leur complétude que lors du travail pandectistique. Ainsi, la propriété privée se construit sous la prémisse du fait que Bello serait, tout comme Portalis, un homme de frontière en ce qui concerne sa conception de propriété. Afin de supprimer ces divergences entre liberté et égalité non résolues par le législateur, nous utiliserons des éléments dogmatiques, normatifs et historiques pour reconstruire la propriété dans un système cohérent tant au niveau de l'objectivité qu'au niveau de la subjectivité.

MotS CLÉs: Propriété privée - Abstraction - Individualisme - Simplification

\section{INTRODUCCIÓN}

La propiedad -o las propiedades- es una de las instituciones de mayor trascendencia, complejidad, discusión y controversia del ámbito filosófico-jurídico, y a pesar de las bibliotecas que se pueden edificar respecto de lo escrito en su entorno, la discusión trasciende la definición y alcanza la posibilidad misma de definirla, todo lo cual no puede sino despertar el interés propio de todo civilista. Sin embargo, de fin de reflejar su alcance, si se le cuestionara a un "mero y vergonzante malabarista de artículos" ${ }^{1}$ respecto a ¿qué es propiedad y cómo caracterizarla?, sin duda de equivocarse respondería que el dominio es un derecho -que aunque acertado resulta parcial-, y lo caracterizaría como derecho absoluto, real, perpetuo y exclusivo -lo cual no es errado, pero no es definitorio de la institución-; y si extendiéramos la pregunta a un jurista propiamente tal, su respuesta variaría según la época y concepción filosófica imperante.

El tema del presente trabajo pertenece a un ámbito de la teoría del derecho de bienes en el contexto del derecho civil, de la teoría general del derecho y de la filosofía política o fundamentación de la propiedad, todo lo cual en consideración a componentes de carácter político, económico, social y jurídico, y no limitado a un mero problema de técnica jurídica. En este marco, se aboca a la ardua tarea de reconstruir la regulación de la propiedad privada -sin desconocer las demás modalidades- que se formula al momento de la codificación mediante la reinterpretación desde los estándares conexos de individualismo, simplificación y abstracción, atingentes a una construcción decimonónica, pero que no alcanzarían su completitud sino hasta la obra pandectista. Se desprende la complejidad de desenmarañar el carácter del Código Civil chileno, y, a fin de dar una respuesta sistémica de la regulación por el derecho nacional, no es posible prescindir del estudio de la regulación otorgada por la Carta Fundamental en el marco de un proceso propio de constitucionalización del derecho civil.

La base del problema es el concepto analítico empleado por el legislador nacional en el artículo 582 del Código Civil, lo cual no obsta a una reinterpretación y denota la pugna de principios libertarios -siempre presente, aunque no

${ }^{1}$ Grossi, Paolo (1992). La propiedad y las propiedades. Un análisis histórico. López, Ángel (Trad.). Madrid: Editorial Civitas, p. 15. 
necesariamente excluyentes- e igualitarios. En otras palabras, esta investigación se aboca al estudio de la propiedad como un problema analítico y normativo con manifiesta incidencia en todo el derecho de bienes y el derecho en general. A este respecto, por motivos atingentes a la laxitud y sistematización del tema de la propiedad, el objeto de estudio debe ser precisado, a fin de dar adecuada respuesta a la interrogante planteada, en torno a tres acápites. El primero es de fundamentación de los estándares propuestos de simplificación, abstracción e individualismo y su necesaria correlación. El segundo y central es analítico y crítico de la reconstrucción intelectual tradicional de la propiedad y su recepción en el Código Civil de Bello, desde los estándares propuestos y sus puntos de tensión, a fin de construir una propuesta de superación. El tercero es una evaluación crítica de las respectivas posiciones construidas a partir de los estándares anteriores.

\section{Fundamentación de LOS ESTÁNDARES PROPUESTOS Y SU NECESARIA CORRELACIÓN}

Este capítulo tiene por objeto dar cuenta de la visión tradicional asignada a la propiedad y servir de fundamento al análisis crítico y propuesta de los acápites siguientes. A este respecto, la relación entre el derecho de bienes y en particular la propiedad y la codificación es inconmensurable, y sólo una vez comprendido el proceso de codificación y las corrientes filosóficas imperantes es dable entender a cabalidad la propiedad como institución atribuiblemente moderna.

La codificación persigue un fin político tendiente a dar unificación a un Estado, así, el código como instrumento emerge como una estructura de poder, y el codificador según la reconstrucción argumentativa de Tarello se cuestionó “... ¿cómo debe ser el derecho? Esta pregunta tuvo, de los sectores más distintos, una respuesta unívoca: el derecho, o mejor las leyes, deben ser simples, claras y pocas, breves, concisas"2; así fue que se requería un derecho cierto y determinado, en donde la codificación es una metodología para expresar el derecho. Ante lo cual surgen dos problemas del ámbito civil: "El primero era la pluralidad de status de los individuos, o pertenencias de clase (...). El segundo era el de la pluralidad de los modos de usufructo de los bienes que eran tipificados por el derecho" ${ }^{3}$. Resulta manifiesto que estos dos problemas, pertenecientes a ámbitos del derecho civil general y derecho de bienes en particular, respectivamente, permiten explicar el primer estándar de simplificación y, consecuencialmente, la abstracción de la propiedad.

En un proceso que no es dable escindir del precedente, la propiedad procedió a abstraerse debido a que el predicado jurídico se redujo y simplificó, por lo que se desligó la propiedad del objeto sobre el cual recae, con lo que se comprende a la propiedad como un derecho en su faz subjetiva. Este proceso de interiorización

\footnotetext{
${ }^{2}$ Tarello, Giovanni (2003). Cultura juridica y política del Derecho. Granada: Comares, p. 48.

${ }^{3}$ Tarello (2003), pp. 48-49.
} 
al fuero interno del sujeto "la propiedad" explica el transito atribuido entre la Edad Media y la propiedad en su concepción moderna, explicando acertadamente instituciones tales como el patrimonio, derecho de garantía general y el carácter elástico de la propiedad por la cual se contrae y se extiende según conste la constitución de un derecho real limitado, que de otra forma debiera entenderse como una desmembración del dominio.

Ahora, ¡cuál es el contenido de la propiedad moderna? Jeremy Waldron nos revela que la propiedad en su filosofía tiene dos clases de problemas: “...problemas analíticos relativos al significado y uso de los conceptos más importantes en el derecho de propiedad, tales como propiedad privada (...) y normativo o justificativo (...) [que buscan responder] ¿Qué justifica dar a las personas derechos de esta naturaleza? Y más específicamente ¿Qué principios justifican la asignación de determinados recursos a determinados propietarios?" ${ }^{4}$. Resulta manifiesto que la interrogante planteada y objeto de análisis se construye en base a un problema analítico, y precisamente la denominada concepción moderna de propiedad definida por abstracción y simplicidad no plasma un contenido normativo o justificativo en particular, y sólo se construye en torno a la formalidad de la propiedad; vale decir, no existe en ella, una apelación a valor alguno de los modelos de justificación, dando cuenta que los estándares condicionan lo que la propiedad es, y aquello que no puede llegar a cubrir.

¿Qué tan individualista, simplificada y abstracta es la propiedad regulada en nuestro ordenamiento jurídico? La respuesta supone definir la controversia difícilmente zanjada entre libertad e igualdad, o, mayormente, entre individualismo y altruismo de la propiedad. Se construyen dos posibles respuestas a la interrogante planteada, a saber, una primera construcción interpretativa, que denominaremos tradicional, que da cuenta de una noción hegemónicamente individualista de la propiedad en la obra del codificador nacional, la cual si bien es objeto de difundida aceptación, igualmente puede ser objeto de los mayores reparos y, consecuencialmente, una segunda construcción reinterpretativa, que denominaremos superadora, que da cuenta de una propiedad con elementos altruistas, desde la concepción del Código Civil de Bello, lo cual se ve mayormente asentado por la injerencia del texto constitucional. Lo anterior, tiene por objeto sistematizar la respuesta a la interrogante planteada sobre el modo - ¿cómo reinterpretar?-, dando cuenta de una noción tradicional y su respectiva construcción crítica mediante ejemplos concretos que reflejan la tensión normativa, y generar una noción de propiedad superadora simplificada y abstracta, en la medida de una noción altruista latente concebible ya en la propia concepción de propiedad del legislador.

${ }^{4}$ Waldron, Jeremy (1996). “Derecho de propiedad”. Soto, Mariano (Trad.), Oxford: Editorial Patterson Dennis, a Companion to Philosophy of Law and Legal Theory Oxford, p. 1. 


\section{ANÁLISIS Y CRÍTICA DE LA RECONSTRUCCIÓN INTELECTUAL}

tradicional de la PROPIEDAD y ReCEPCión en el Código Civil de Bello

“HACIA LOS ESTÁNDARES” PROPUESTOS

El punto de partida del problema de la propiedad en nuestro sistema civil es que nuestro legislador procede a definir la propiedad en términos analíticos, similar a lo previsto en Código Napoleónico, mediante una cuantificación de atributos, por lo que el propietario no es más que quien usa, goza y dispone de una cosa, lo cual ha llegado a calificar a propósito del Code como un régimen de propiedad que comparte elementos modernos, “....sin embargo no ha cancelado completamente la tradicional articulación del dominio en un ius disponiendi y en un ius abutendi fruto de una cultura diversa y de una visión objetiva de la pertenencia..." ${ }^{\text {. Es ne- }}$ cesario precisar que la definición mediante el empleo de un conjunto de facultades no es formal, sino sustancial; definir en base a facultades e identificar al propietario como quien usa, goza y dispone resulta carente de contenido, siendo evidente que la propiedad resulta ser algo más que dichos u otros atributos, verbigracia, exclusividad, y se constituye en una unidad. La posible respuesta es que la propiedad puede ser reinterpretada en relación a la comprensión de tres categorías de estándares, a razón el individualismo, la abstracción y la simplificación, superando el prisma individualista carente de sentido histórico y de realidad normativa y coherentemente con ello determinando el alcance de la simplicidad a la injerencia de derechos reales limitativos y no a un contenido retributivo. Y, finalmente, en cuanto a la abstracción, la reinterpretación supone corregir sistémicamente su alcance y no limitar la concepción del derecho de propiedad a una faz subjetiva, respecto de todo lo cual se procederá a dar los fundamentos a continuación.

\subsection{Tratamiento del estándar del individualismo posesorio}

Este elemento debe comprenderse que atiende al rendimiento explicativo para entender la propiedad, edificando "un sistema en que la libertad individual es concedida y regulada como un fin en sí misma, por fuera de cualquier subordinación a los intereses del grupo" 6 , siendo una reconstrucción intelectual mediante la cosmovisión lockeana de propiedad, extendida en la sociedad burguesa-liberal, alejada en principio de la comprensión revolucionaria que reconoce la pugna entre libertad e igualdad.

La posición adoptada es una visión superadora del estándar del individualismo, con incidencia directa en la comprensión de la abstracción y en especial de la simplificación. La base de la construcción superadora hacia la post-modernidad

\footnotetext{
5 Grossi (1992), pp. 125-126.

${ }^{6}$ De Martino, Francesco (2005). Individualismo y derecho romano privado. Hinestrosa, Fernando (Trad.), Bogotá: Editorial Universidad Externado de Colombia, p. 16.
} 
supone, en primer término, pasar una pequeña revista por la disputa de fondo. La interpretación clásica se ha atribuido un carácter individualista, fundado en que "El código civil chileno al igual que su par francés exalta el Individualismo, mediante la consagración de normas de derecho privado de la libertad, la igualdad y la voluntad..." , ya que resulta evidente que en materia de propiedad "Bello quiso introducir, en el espíritu de la legislación, el principio de libertad, tan característico del ideario de la Revolución Francesa (...) Bello incorpora a la legislación el concepto de propiedad proveniente del iusnaturalismo racionalista" ${ }^{\prime}$. Sin embargo, la interpretación tradicional puede ser superada en un intento interpretativo desde el Código Civil, ya que en él conviven y sin solución la dicotomía libertad-igualdad como actitudes opuestas, y que la problemática trasciende lo jurídico y se adentra en lo político y moral, ya que "El problema reside en que la moralidad no es ya unívocamente altruista hay un conflicto de modalidades. Tampoco es ya la política inequívocamente individualista hay argumentos a favor del colectivismo, la regulación, el Estado benefactor, junto con la teoría del desarrollo económico a través del laissez-faire" ${ }^{\prime}$, de lo cual es dable afirmar que la moralidad y política están presentes, pero no es posible atribuírselas a una posición determinada.

En segundo término, desde un punto de vista analítico, la reinterpretación puede ser fundada en razones históricas y normativas. En cuanto a las razones históricas, surge el primer elemento a razón de la oscuridad de las fuentes empleadas por el legislador ya que “... en ninguna parte Bello explicita su concepto de propiedad, ni señala las fuentes a que recurrió para elaborarlo. Solo hay indicio tangenciales al respecto en sus intervenciones en el Senado cuando se discute la ley de ex vinculación de mayorazgos, donde insiste en la idea de un dominio pleno, perfecto y absoluto"10; es claro que estas últimas remisiones dicen relación expresa con la libre circulación de la riqueza respecto de los grupos familiares acaudalados y no la carencia de contenido altruista social. El segundo elemento es la controvertida lectura de los historiadores del derecho de la interpretación del Código Napoleónico, siguiendo en este punto a Alfons Burge, para el cual "los representantes de la nueva concepción liberal de la propiedad en la doctrina francesa, no llegarían a ésta a través de una exegesis del artículo 544, sino que por la vía de introducir en

\footnotetext{
7 Tapia Rodríguez, Mauricio (2005). Código Civil 1855-2005. Evolución y perspectivas. Santiago: Editorial Jurídica de Chile, p. 36.

8 Soza Ried, María de los Ángeles (2004). "La influencia del Código Civil francés en el derecho civil chileno en materia de bienes y propiedad". Cuadernos de extensión Universidad de Los Andes, Facultad de Derecho, No 9, p. 76.

9 Kennedy Duncan (2001). "Forma y sustancia en la adjudicación del derecho privado". Holguín, Magdalena y Escobar, Alicia (Trad.). En: Sociología Jurídica: Teoría y Sociología del Derecho en Estados Unidos, García, Mauricio (Ed.), Bogotá, Universidad Nacional de Colombia, p. 171.

10 Brahm García, Enrique (1996). "El concepto de propiedad en el Código Napoleónico. Una nueva interpretación de su artículo 544 en la historiografía jurídica alemana". Revista Chilena de Derecho, Vol. 23, No 1, p. 11.
} 
esa norma la filosofía idealista y liberal kantiana tal cual había sido integrada al sistema de Savigny" ${ }^{11}$, lo cual es manifiestamente aplicable al Código Civil chileno al contemplar limitaciones.

En cuanto a las razones normativas, resulta manifiesto e innegable en principio el imperio de una libertad como fundamento de la propiedad, pero ello no obsta a la contemplación de limitaciones tendientes a una retribución. En tal sentido, es la propia definición legal en el artículo 582 del Código Civil, por la cual "El dominio (que se llama también propiedad) es el derecho real en una cosa corporal, para gozar y disponer de ella arbitrariamente; no siendo contra la ley o contra derecho ajeno" (la cursiva es propia), además de normas diseminadas, tales como el artículo $945 \mathrm{del}$ Código Civil respecto de cavar el pozo y privar de agua al vecino sin provecho, que aun cuando derogado por razones de sistematización normativa se encuentra vigente en el Código de Aguas. Estas y otras normas, más que constituir excepciones denotan precisamente tensiones con el individualismo en la formulación y consagración de la propiedad, lo cual da cuenta de la conformación de teorías desde el Código, tales como la teoría de la inmisión y la teoría del abuso del derecho, que confluyen con fundamentos alejados del individualismo. En cuanto a esto último, dichas tensiones deben ser explicadas ante la carencia de un concepto legal de propiedad y la constante disputa en su justificación entre los principios de libertad e igualdad; de ello se desprende que la sola posibilidad de abusar de un derecho se construye sobre bases distintas de la concepción liberal y exclusivamente subjetivista de los derechos, y en particular, del derecho de propiedad.

La remisión a la teoría de la inmisión no tiene por objeto redundar en una reiteración inocua de la doctrina especializada, sino dar cuenta de su relación directa con el tema objeto de estudio a fin de reinterpretarlo adecuadamente. El fundamento de la teoría de la inmisión descansa en la comprensión de la propiedad en tensión con el individualismo, y da cuenta de un carácter absoluto graduable y, por ende, no definible de la propiedad. Debemos reconocer la postura de Burge, que señala "el artículo 544, de la manera más absoluta [nuestro arbitrariamente], habría perseguido distinguir claramente el dominio del usufructo (...) En su origen no pretendió el legislador francés establecer un derecho absoluto, exclusivo y perpetuo, sino solo terminar con las estructuras propietarias del antiguo régimen, pero autorizando la intervención del estado bajo el signo de la igualdad"12; todo lo cual resulta de manifiesto al contemplar los límites de la propia norma nacional en propiedad, en cuanto a la ley y derecho ajeno. Surge la interrogante: ¡es la teoría de la inmisión una excepción a la consagración individualista? El supuesto de aplicación es que $\mathrm{A}$ en el ejercicio de actos propios del dominio derive consecuencias, tales como, ruidos o contaminación, las cuales penetran en la esfera de

11 BRAHM (1996), p. 10.

12 Brahm (1996), p. 7. 
un bien ajeno cuyo titular es $\mathrm{B}$, lo que supone plantearse el problema desde una doble perspectiva. En primer término, resulta manifiesto que para efectos de B la teoría no es novedosa, ya que representa el ejercicio arbitrario de su propiedad sin molestia de tercero, dado que "Lo interesante de la teoría de la immissio es que establece que los actos que perjudican la posesión de un bien, para poder ejecutarse, deben estar amparados por una servidumbre"13. Ahora, desde la perspectiva de A, se manifiesta una tensión con la aplicación clásica del individualismo, que pugna con la aplicación en consideración a los derechos del tercero.

Por último, a efectos de dar una respuesta sistémica e integral a la interrogante planteada en nuestro ordenamiento jurídico a razón del individualismo no puede obviarse el tratamiento que recibe la propiedad a nivel constitucional, en particular, en cuanto a la función social ${ }^{14}$, institución que irrefutablemente da cuenta del altruismo igualitario. De la mera lectura de las normas del texto constitucional directamente estatuidas se desprende que la función social es constitutiva de limitaciones y obligaciones al derecho de propiedad en un sentido subjetivo. Es necesario precisar que la concepción de propiedad carente de contenido o límites sociales no sólo puede ser atribuida al individualismo, sino que responde al estándar de la abstracción de la propiedad mediante la interiorización de la propiedad en el sujeto, cuestión que es dable explicar ante la carencia de un compromiso legal por un concepto justificativo de propiedad, impropio de una institución social y, en particular, de la propiedad privada ya que “... buscamos una justificación para la propiedad privada, porque ella priva a la comunidad del control de los recursos que pueden ser de importancia para el bienestar de sus miembros, y especialmente porque requiere que nosotros movilicemos la fuerza social en apoyo a la exclusión de muchos miembros de nuestra sociedad sobre todos y cada uno de los usos de los recursos qué se necesitan para vivir" ${ }^{15}$.

No es el punto el estudio detallado de elementos normativos de la regulación constitucional de propiedad, sino dar cuenta de la reconstrucción manifiesta del estándar de propiedad realizada por el constituyente, ya que a la sabida concepción liberal le otorga un condimento de su esencia como es el contenido social, lo cual, aunque paradigmático, resulta ser realizado en términos cercanos a la reforma constitucional del año 1967. En cambio, la cuestión de fondo es que la función social es un punto crítico de la construcción individualista de la propiedad, al contemplar una fórmula más integradora del derecho de propiedad en la sociedad, la construcción meramente subjetiva del derecho de propiedad en su contenido interno resulta cuestionada, ya que "La enseñanza que podemos obtener de algu-

\footnotetext{
13 Amunátegui Perelló, Carlos (2009). "No siendo contra derecho ajeno: Hacia la formulación de una teoría de las inmisiones en nuestro Código Civil”. Revista Chilena de Derecho, Vol. 36, No 3, p. 515.

${ }^{14}$ Véase artículo 19 No 24 inciso $2^{\circ}$ Constitución Política de la República.

15 WALDRON (1996), p. 7.
} 
nas investigaciones, es que, una construcción de propiedad totalmente adherida al paradigma de derecho subjetivo solo es posible a costa de un total sacrificio de la función social" ${ }^{16}$.

Se desprende con claridad la reinterpretación sostenida, desde una concepción de propiedad en principio inobjetablemente individualista del Código Civil, entorno a definiciones, normas, teorías e historia que dan cuenta de un contenido al menos abierto y con mayor riqueza retributiva, ya que en tal término “...la jurisprudencia y, desde luego, el legislador, están facultados para conducir la institución mediante estas restricciones [ley y derecho ajeno] para que cumpla adecuada función; en esos términos, estimamos que el concepto es abierto y flexible"17.

De la abundante fundamentación a la proposición sostenida, cabe sostener una objeción en el marco estricto de la interrogante planteada en el ámbito del Código Civil, ya que atender al individualismo a la luz de la Carta fundamental resulta desacertado. Aquel marco se funda en razones históricas, atendiendo a la época de dictación del Código Civil y el imperio de la libertad como principio dominante a la luz de la expresión arbitrario, posición dominante en la doctrina, que ya ha sido objeto de crítica precedentemente, aun cuando la voz arbitrario debe entenderse remitido a la injerencia de terceros sin derechos reales constituidos. Ahora, todo lo anterior merece un comentario final: el individualismo como objeto de estudio no significa obviar ni yuxtaponer a la comprensión individualista propia del humanismo, por la cual el individuo es el centro y la legislación debe tener como eje al sujeto, cuestión distinta es la prescindencia de la consideración de los demás, y la consideración del Código del individuo en forma abstracta, por lo cual la propiedad ya no se identificó con la cosa, sino que el individuo se cosificó, así "La cosa -como enseña el príncipe de los Pandectistas Bernhard Windcheid- es sólo un fragmento de la naturaleza irracional. La propiedad moderna está construida sobre el individuo y para el individuo" 18 .

\subsection{Tratamiento del estándar de abstracción}

En relación al estándar de abstracción, su alcance es latente en el Código Civil de Bello, lo que se refleja en los artículos 582, 583, 889, y con genialidad sistémica en la estructura del patrimonio como atributo de la personalidad en la teoría clásica, todo lo cual permite explicar que un mendigo o sujeto carente de bienes pueda ser susceptible de relaciones patrimoniales; vale decir, el objeto fue

\footnotetext{
${ }^{16}$ Rodota, Stefano (1986). “El terrible derecho”. Díez-Picazo, Luis (Trad.). En: Estudios sobre la propiedad privada, Madrid: Editorial Civitas, p. 232.

17 Peñailillo Arévalo, Daniel (2007). "Problemas antiguos y nuevos en materia de derechos reales". En: El Código Civil de Chile (1855-2005), Santiago: LexisNexis, p. 444.

${ }_{18}$ Grossi, Paolo (2004). "Propiedad y contrato". En: El Estado Moderno en Europa: Instituciones y Derecho, Madrid: Trotta, p. 131.
} 
abstraído y diferenciado del derecho que en aquél recae. Lo anterior, supone la concepción de propiedad en forma unitaria, y se ve reflejada la abstracción en su máximo alcance en el artículo 2465 del Código Civil, en cuanto "Toda obligación personal da al acreedor el derecho de perseguir su ejecución sobre todos los bienes raíces o muebles del deudor, sean presentes o futuros, exceptuándose solamente los no embargables, designados en el artículo1618" (la cursiva es propia). Ello no obsta a desconocer que Bello al igual que su par Portalis es un hombre de frontera, tal como se caracteriza al Código Napoleónico: "dos almas, dos mentalidades, dos escrituras; no hay dudas de que los compiladores del Código y del texto del artículo [544 del Code, semejante mas no idéntico al 582 CC chileno] se han embriagado por la retórica decimonónica de la nueva propiedad liberada..." ${ }^{19}$. En este marco, si bien la abstracción como técnica de codificación resulta innegable, cuestión diversa es desconocer las dificultades y puntos conflictivos en la constitución de la propiedad abstracta en nuestro sistema, los que se proceden a desarrollar a continuación.

En primer término, se proponen dos problemas sistémicos claros de la propiedad regulada en el Código Civil que dicen relación con la recepción del legislador. El primero de ellos ya se encuentra desarrollado: la noción analítica de propiedad, por lo que me remito a lo señalado. El segundo problema, y relacionado con el anterior, dice relación con el tratamiento que reciben los derechos reales limitados por nuestro legislador, derechos que no confieren todas y cada una de las facultades propias del dominio y que son susceptible de discusión ya desde su propia denominación en torno al carácter de derechos reales limitados o meras desmembraciones del dominio, lo cual da cuenta de esta doble alma por ser un código de frontera en relación a la constitución de los derechos reales limitados.

La constitución de los derechos reales limitados goza en principio de una dicotomía sistémica entre el Código Civil y el Reglamento del Conservador de Bienes Raíces, ya que este último cuerpo de leyes procede a distinguir "entre tradición de un derecho real (ya constituido) y la constitución (efectuada por el propietario) del mismo (art. 52 No 1 y 2 respectivamente)" ${ }^{20}$, cuestión que merece dos comentarios. El primero dice relación con que la distinción propuesta en el reglamento se condice con una propiedad abstracta y unitaria propia de la modernidad, y además que el legislador en este punto se distanció de la noción moderna de propiedad y se remitió indistintamente a tradición, tal como se da cuenta en el derecho real de usufructo a partir del artículo 767 del Código Civil, del cual se desprende en el ámbito de su constitución y solemnidad por acto voluntario entre vivos de carácter inmueble que su reconocida controversia doctrinaria es respecto del rol de la inscripción, en cuanto al rol de solemnidad o requisito del acto, mas no en cuanto a su carácter de tradición. Sin embargo, una propiedad abstracta de

${ }^{19}$ Grossi (1992), p. 125.

${ }^{20}$ Peñailillo Arévalo, Daniel (2006). Los bienes. La propiedad y otros derechos reales. Santiago: Editorial Jurídica de Chile, cuarta edición. 
propiedad exige la diferencia entre constitución y tradición, ya que la propiedad es unitaria y elástica.

En este ámbito, da cuenta de la confusión legislativa el tratamiento asistémico que recibe el derecho de uso y habitación; a este respecto, el artículo 819 del Código Civil sostiene su carácter intransferible. Sin embargo, y en manifiesta incoherencia, el artículo 686 del mismo cuerpo de leyes procede a exigir la inscripción para la tradición del derecho de uso y habitación. Este último tratamiento puede ser explicado a luz de una doctrina tradicional mediante un error de técnica legislativa, o una visión sistémica incoherente y en pugna de la tensión no resuelta en el tratamiento de la propiedad. Y, finalmente, en cuanto al fideicomiso resulta en principio coherente con la noción abstracta de propiedad al aludir a la constitución y no a la tradición en el artículo 735 del Código Civil, y en el mismo sentido el artículo 52 No 2 Reglamento del Conservador de Bienes Raíces. Sin embargo, la interpretación de la doctrina da cuenta de la misma incoherencia sistémica, a saber "cuando se constituye por testamento es claro que la inscripción no significa tradición de la propiedad fiduciaria, porque el modo de adquisición es allí la sucesión por causa de muerte. (...) cuando se constituye por acto entre vivos, la inscripción tiene rol de tradición de la propiedad fiduciaria..." ${ }^{21}$, como se puede apreciar resulta manifiesto que, pese al tenor literal de la ley, la noción interpretativa imperante es la desmembración del dominio, lo cual no se condice con una propiedad abstracta, y da cuenta de la posibilidad de una reformulación de la interpretación.

En segundo término, se propone una crítica sistémica asumiendo la abstracción latente en el Código Civil en su concepción burguesa respecto del tratamiento en el ordenamiento jurídico nacional de la propiedad, ya que se limita a explicar a la propiedad como derecho en su faz subjetiva, pero carece la abstracción de capacidad para dar cuenta de la propiedad en su faz objetiva, es que “... no se distinguen los distintos planos que puede tener la propiedad, ya sea como régimen jurídico (plano objetivo) o como derecho (plano subjetivo)"22. Aquí se expresa con plenitud una cuestión de fondo: la propiedad es insoslayablemente un derecho subjetivo, situado desde el titular en su perspectiva individual, pero no se pueden desconocer los intereses de la colectividad, expresados a nivel constitucional en la denominada función social de la propiedad, aun cuando resulte evidente que aquella visión no puede invadir el núcleo individual, mutando al titular en un gestor de cuestiones ajenas.

En tercer término, se propone dar cuenta de la interrelación crítica a la construcción clásica de patrimonio en relación manifiesta a una crítica solapada a las limitaciones de la concepción abstracta de la propiedad; así, en cuanto a nivel explicativo del patrimonio "... La omnipotencia de la voluntad del hombre le

\footnotetext{
${ }^{21}$ Peñailillo Arévalo (2006), p. 195.

22 Cordero Quinzacara, Eduardo (2006). "La dogmática constitucional de la propiedad en el derecho chileno". Revista de derecho (Valdivia), Vol. 19, No 1, p. 128.
} 
permite reunir derechos en un todo. Semejante afirmación no sorprende por parte de autores de la escuela liberal, que hacían descansar el derecho sobre la persona y su voluntad" 23 , de lo cual resulta manifiesto que la teoría clásica es limitada al momento de explicar los patrimonios de afectación, y vacía al suponer que sin bienes (contenido) dispone de patrimonio (continente), emergiendo como explicación manifiesta la abstracción. Sin embargo, resulta evidente que la teoría del patrimonio permite explicar instituciones abstractas, tales como el derecho de garantía general del artículo 2465 del Código Civil. Respecto de este último, ¿̇resulta perniciosa su concepción que permite el endeudamiento más allá de la capacidad económica del deudor? En principio la respuesta debe ser negativa, ya que atiende al principio de libre circulación de la riqueza, esto por el hecho de responder con bienes presentes y futuros, permite no entroncar los bienes existentes al momento de la contratación a fin de responder por la acreencia, aun cuando ello no significa desconocer el carácter eventualmente vacío por carecer de bienes e incurrir en un sobreendeudamiento, pero respecto de lo cual debe ser enmendado mediante una política económica acorde.

Sumados, de los puntos de inflexión descritos emerge el estado de la cuestión por el cual la propiedad del Código Civil no es plenamente abstracta, lo cual permite una base normativa tendiente a una reformulación, ya que la abstracción -sin afán de desconocer su ámbito de vigencia- sería una construcción plenamente obtenida recién en la época pandectista a propósito de la concepción de propiedad. Ahora, ¿cómo se debe reinterpretar? El punto de partida es descartar un estándar de pertenencia material concretizando la propiedad, ya que daría cuenta de un proceso de cese de la libre circulación de la riqueza al caer en desuso instituciones, tales como el derecho de garantía general y no la pertenencia en sí, ya que "La propiedad de bienes es una suerte de decantación o precipitado particular de un espacio intencional vinculado con un proyecto del hombre (...). El carácter de enajenabilidad muestra a la propiedad de bienes de algún modo como la más débil de las relaciones de pertenencia fundadas por los espacios intencionales. La explicación de ello reside probablemente en el carácter histórico que, aun desde un punto de vista individual, poseen las necesidades humanas que dan origen a los proyectos dentro de los cuales dichos bienes se muestran como propios" 24 . Así las cosas, la respuesta a la disyuntiva planteada entre un estándar moderno y post-moderno, explicado en párrafos anteriores, no responde acertadamente a la pregunta originaria tendiente a la reformulación de la propiedad según el estándar de la abstracción, sino que se plantea comprender el alcance de la abstracción.

${ }^{23}$ Lyon Puelma, Alberto (1993). Teoría de la personalidad: personas naturales, personas juridicas. Santiago: Ediciones Universidad Católica de Chile, p. 214.

${ }^{24}$ BARCEló, Joaquín (1993). "Acerca del fundamento del derecho de propiedad”. Estudios Públicos, No 52, p. 273. 
En cuanto a las objeciones que pudiera formularse a dicha posición son principalmente las ya explicadas: subjetivismo y la unidad consecuenciales a la abstracción. Sin embargo, y más allá del juicio crítico propuesto, la abstracción explica acertadamente la propiedad desde la perspectiva subjetiva, pero no obsta a la fundamentación de la propiedad desde su ámbito objetivo, sino, y más bien, se debe a una comprensión dicotómica individualismo-altruismo. Y, finalmente, la abstracción permite explicar la unidad de la propiedad al adentrarse en el yo del sujeto, pero no obsta a la proliferación de propiedades, ya que lo que explica la abstracción es un núcleo como derecho, y no necesariamente su identidad, verbigracia, piénsese en cualquier ordenamiento que consagre la propiedad abstracta como el Código Civil y Comercial Argentino ${ }^{25}$, el cual de ninguna manera significa desconocer el contenido social y propiedades especiales, tales como la industrial. Un contraargumento plausible es la posibilidad cierta de definir propiedad como núcleo unitario, ante la proliferación de propiedades, sino en cuanto a derecho, cuestión que resulta de interés para otro tema de investigación.

\subsection{Tratamiento estándar de simplificación}

En relación al estándar de simplificación no requiere mayor detenimiento el hecho de que la propiedad regulada en el Código Civil se ha simplificado, la pregunta de fondo es determinar cuál es el alcance de dicho proceso simplificador. En otras palabras, su finalidad era limitar el contenido retributivo absolutizando la propiedad respecto de un contenido social o meramente limitar las cargas impuestas al dominio por terceros permitiendo la libre circulación de la riqueza. La cuestión de fondo es de difícil resolución, y resulta evidente la injerencia de la posición adoptada por el legislador respecto del espíritu individualista, siendo de importancia el rol facilitador de la simplificación en un carácter funcional de la libre circulación de la riqueza en sus diversas formas contractuales.

El proceso de simplificación en nuestro Código Civil resulta manifiesto en cuanto a los sujetos, puede observarse de la diada constituida por la definición y tratamiento de la persona natural y persona jurídica, contemplada en los artículos 55 y 545 del Código de Bello, respectivamente. En cuanto a la simplificación del objeto es de suma complejidad, pero en el fondo la disputa es ideológica entorno a la concepción individualista dado su carácter instrumental. En el marco de este proceso la propiedad fue objeto de reducción al menos de las innumerables limitaciones al dominio, que se sintetiza en el principio de libre circulación de la riqueza, que es precisamente reacción por contraposición a la entrabada cosmovi-

${ }^{25}$ Código Civil y Comercial de la Nación Argentina, artículo 1941. "Dominio perfecto. El dominio perfecto es el derecho real que otorga todas las facultades de usar, gozar y disponer material y jurídicamente de una cosa, dentro de los límites previstos por la ley. El dominio se presume perfecto hasta que se pruebe lo contrario". 
sión de la propiedad durante la edad media, por lo que en si es una manifestación del individualismo frente al corporativismo de la época feudal.

El mentado carácter instrumental de la simplificación de la propiedad no se traduce en que sea inocua a la disputa no resuelta entre individualismo y altruismo, ya que "La simplicidad atestigua la elección de librar al dominium de las variaciones de lo contingente para absolutizarlo en el ámbito del sujeto, insertándolo lo más posible en su interior" ${ }^{26}$, pero carece de capacidad explicativa del individualismo, sino, y por el contrario, es la simplificación que responde al mayor o menor grado de individualismo posesorio. De ello, su carácter manifiestamente instrumental y el alcance expuesto de un individualismo sobredimensionado, es que la simplificación responde a un estándar acorde a la propiedad actual, y en cuanto a objeciones debe remitirse a lo expresado a propósito del individualismo.

En síntesis, y sin afán de reduccionismo de la problemática de la propiedad, ¿cómo reinterpretar la propiedad? Esta emerge en su modernidad abstracta como derecho subjetivo desprovista de la pertenencia material y adentrada en el "yo" del sujeto capaz, simplificado, careciendo de límites externos que permiten la libre circulación de la riqueza, e individualista, carente de sentido retributivo. Sin embargo, pese a la tesis imperante, resulta plausible una relectura desde el propio Código Civil que da cuenta de los puntos de tensión más o menos graduables de la modernidad de la propiedad, y prescindir de todo contexto histórico cultural al sostener un individualismo desprovisto de altruismo resulta ser una concepción, aunque plausible, carente de sentido de realidad, aún más si se estima el tratamiento sistémico de la propiedad en nuestro sistema.

\section{EVALUACiÓN CRÍTICA DE LAS RESPECTIVAS POSICIONES CONSTRUIDAS A PARTIR DE LOS ESTÁNDARES PROPUESTOS}

Resulta manifiesto que la posición tradicional interpretada a la luz de los estándares de abstracción, individualismo y simplificación fue objeto de una evaluación crítica, a fin de determinar sus puntos de tensión en el sistema de propiedad nacional y dar cuenta de la posibilidad cierta de una reinterpretación superadora. De lo anterior se desprende que, cualquier evaluación crítica resulta inocua y reiterativa de lo expuesto, y da cuenta de la tensión latente e imperante desde la cosmovisión del propio legislador.

La configuración crítica de la visión tradicional supone una nueva perspectiva, como resulta ser la visión superadora o post-moderna, la cual no es ajena a una evaluación crítica, pese a lo imperante en consideración de la regulación sistémica de propiedad en nuestro ordenamiento jurídico nacional. La construcción argumentativa, histórica y normativa propuesta y desarrollada resulta ser objeto de controversia, lo cual en todo caso no constituye novedad alguna en consideración

${ }^{26}$ Grossi (1992), p. 110. 
a las pasiones que despierta la propiedad, teniendo presente la necesidad de justificación en dos perspectivas: “... Primero, necesitamos justificar la idea general de tener cosas bajo el control de sujetos privados. En segundo lugar, debemos justificar los principios en virtud de los cuales algunos llegan a ser propietarios de determinados recursos mientras otros no" 27 .

En primer término, una visión crítica a la noción abstracta de propiedad recogida en nuestro Código Civil no da cuenta de una construcción autónoma o superadora que integre el sistema de derecho civil y dé fundamento a instituciones tales como el derecho de prenda general. El ejemplo precedente no es inocuo, sino que da cuenta del alcance filosófico de la concepción de propiedad abstracta en nuestro sistema jurídico, ya que pese a la falta de sistematicidad en su regulación, no es dable concluir una propiedad post-moderna eventualmente concreta, cuya raigambre de pertenencia material ha sido superada por 300 años de historia. La abstracción de la propiedad permite explicar instituciones tendientes a la libre circulación de la riqueza, que sin mediar aquélla se vería entrampada, por ejemplo, al deber garantizar con bienes presentes un crédito. La regulación en nuestro sistema jurídico es susceptible de un análisis diferenciado, siendo la abstracción cuestionable en el ámbito de la propiedad al sólo dar cuenta de su ámbito subjetivo, pero que resulta incuestionable como estándar del sistema de derecho civil en general.

Ahora, en cuanto al estándar del individualismo post-moderno o posición superadora en torno a la edificación de un principio de altruismo iluminador de la propiedad, resulta de suma complejidad dar cuenta de una evaluación crítica en cuanto al fondo planteado, al erigir el derecho de propiedad como un derecho dual, vale decir, como derecho con contenido objetivo y subjetivo, más allá de la necesidad de equilibrar a fin de no volver al propietario en un gestor de negocios ajenos de carácter estatal. Sin embargo, puede ser objeto de una evaluación crítica la reformulación interpretativa ajena al estándar individualista, en base a una supuesta interpretación progresiva del Código Civil fundada en una comprensión histórica-filosófica individualista del legislador originario.

En cuanto a la evaluación crítica del estándar de simplificación, resulta manifiesto de lo expuesto que en su carácter instrumental atribuido será objeto de crítica según el sentido y alcance que se le otorgue a razón de su extensión, por lo que me remito a lo dicho precedentemente en cuanto a la abstracción y particularmente al individualismo, ya que "La relativización de los derechos subjetivos en vista de la función social tendrá grandes consecuencias en la dogmática jurídico-civil, con una mayor o menor repercusión en el ámbito constitucional. En el ámbito estrictamente civil retorna con particular fuerza la doctrina del abuso del derecho en lo que se ha llamado su manifestación objetiva"28.

\footnotetext{
27 WaLdRON (1996), p. 9.

${ }^{28}$ Cordero Quinzacara, Eduardo (2008). "De la propiedad a las propiedades: La evolución de la concepción liberal de la propiedad”. Revista de Derecho (Valparaíso), Vol. 31, No 2, p. 512.
} 
Finalmente, los estándares pueden y deben ser interrelacionados desde una perspectiva histórica y justificativa; sin embargo, difieren manifiestamente en su rol. En cuanto a la abstracción de la propiedad, es miope frente a la faz objetiva del dominio y se relaciona con el individualismo en el siguiente sentido "... A la abstracción de la propiedad corresponde la abstracción del sujeto, y sólo esto hace posible la transformación del individualismo posesivo originario en una forma general de organización de la sociedad: la sociedad de los propietarios libres e iguales" 29 , y por su parte, la simplificación es funcional a la codificación. Atendiendo a la naturaleza misma de la controversia y a la falta de definición legislativa, el contenido normativo o justificativo de la propiedad resulta objetable, aunque manifiestamente no es meramente individualista ya para el propio codificador.

A MODO DE CONCLUSIÓN: APORTACIONES PARA UN DEBATE INCONCLUSO

1. El Código Civil chileno es un código de frontera, al igual que su par francés, en cuanto a la constitución de la propiedad moderna, por lo que la abstracción, simplicidad e individualismo no responden con plenitud al estándar de la propiedad, y con menor razón aún, al estándar de una propiedad "post-moderna".

2. La base del problema es el concepto analítico de propiedad impropio de la propiedad moderna y la expresión arbitraria empleada por el legislador, que ha sido interpretada de individualista carente de contenido igualitario-altruista, aun cuando se desconocen los propios límites que la noción contempla y que se expresan en normas diseminadas en el Código Civil, tensión que se refleja en doctrinas, tales como la teoría de la inmisión.

3. La comprensión del derecho, del derecho civil y en particular del derecho de bienes no resulta posible sin la comprensión de la propiedad, más aún, la propiedad no resulta comprensible sin la comprensión de la discusión filosófico-normativa en torno a ella.

4. La simplificación actuó como instrumento de codificación en cuanto a sujeto y predicado jurídico limitado a las trabas de la libre circulación de la riqueza mediante el excesivo empleo de derechos reales limitados de dominio, mas no simplificó el contenido social de la propiedad, salvo una postura individualista.

5. La abstracción fundamenta la propiedad como un derecho en su carácter subjetivo, pero es miope a la concepción de propiedad como derecho objetivo, aun cuando no la niega, y, por ende, su recepción incide el alcance limitado o no del estándar del individualismo.

6. El estándar del individualismo carente de un sentido de realidad puede ser superado ya desde el estudio del propio Código Civil, y no admite mayor discusión en revisión sistémica del ordenamiento jurídico nacional. De ello se desprende que

${ }^{29}$ Barcellona, Pietro (1996). El individualismo propietario. Madrid: Comares, p. 46. 
la reinterpretación propuesta de la propiedad supone una abstracción y simplificación carente de un sentido individualista.

\section{BIBLIOGRAFÍA CITADA}

Amunátegui Perelló, Carlos (2009). "No siendo contra derecho ajeno: Hacia la formulación de una teoría de las inmisiones en nuestro Código Civil”. Revista Chilena de Derecho, Vol. 36, No 3, pp. 505-525.

Barcellona, Pietro (1996). El individualismo propietario. Madrid: Comares.

BARCELÓ, Joaquín (1993). "Acerca del fundamento del derecho de propiedad". Estudios Públicos, No 52, pp. 247-275.

Brahm García, Enrique (1996). "El concepto de propiedad en el Código Napoleónico. Una nueva interpretación de su artículo 544 en la historiografía jurídica alemana”. Revista Chilena de Derecho, Vol. 23, No 1, pp. 7-12.

Cordero Quinzacara, Eduardo (2006). "La dogmática constitucional de la propiedad en el derecho chileno". Revista de Derecho (Valdivia), Vol. 19, No 1, pp. 125-148.

Cordero Quinzacara, Eduardo (2008). "De la propiedad a las propiedades: La evolución de la concepción liberal de la propiedad”. Revista de Derecho (Valparaíso), Vol. 31, No 2, pp. 493-525.

De Martino, Francesco (2005). Individualismo y derecho romano privado. Hinestrosa, Fernando (Trad.), Bogotá: Editorial Universidad Externado de Colombia.

Grossi, Paolo (1992). La propiedad y las propiedades. Un análisis histórico. López, Ángel (Trad.). Madrid: Editorial Civitas.

Grossi, Paolo (2004). "Propiedad y contrato". En: El Estado Moderno en Europa: Instituciones y Derecho, Madrid: Trotta, pp. 127-136.

Kennedy Duncan (2001). "Forma y sustancia en la adjudicación del derecho privado”. Holguín, Magdalena y Escobar, Alicia (Trad.). En: Sociología Juridica: Teoría y Sociología del Derecho en Estados Unidos, García, Mauricio (Ed.), Bogotá: Universidad Nacional de Colombia, pp. 163-187.

Lyon Puelma, Alberto (1993). Teoría de la personalidad: personas naturales, personas jurídicas. Santiago: Ediciones Universidad Católica de Chile.

Peñailillo Arévalo, Daniel (2006). Los bienes. La propiedad y otros derechos reales. Santiago: Editorial Jurídica de Chile, cuarta edición.

Peñailillo Arévalo, Daniel (2007). "Problemas antiguos y nuevos en materia de derechos reales”. En: El Código Civil de Chile (1855-2005), Santiago: LexisNexis, pp. 429-473.

Rodota, Stefano (1986). "El terrible derecho". Díez-Picazo, Luis (Trad.). En: Estudios sobre la propiedad privada, Madrid: Editorial Civitas. 
Soza Ried, María de los Ángeles (2004). "La influencia del Código Civil francés en el derecho civil chileno en materia de bienes y propiedad". Cuadernos de extensión Universidad de Los Andes, Facultad de Derecho, No 9, pp. 69-79.

Tapia Rodríguez, Mauricio (2005). Código Civil 1855-2005. Evolución y perspectivas. Santiago: Editorial Jurídica de Chile.

Tarello, Giovanni (2003). Cultura jurídica y politica del Derecho. Granada: Comares. WaLdRon, Jeremy (1996). "Derecho de propiedad". Soto, Mariano (Trad.), Oxford: Editorial Patterson Dennis, a Companion to Philosophy of Law and Legal Theory Oxford, pp. 1-34. 